\title{
Das Mittel zur Suizidbeihilfe und das Recht auf den eigenen Tod
}

\author{
Das Bundesgericht verneint im BGE 133158 vom 3. November 2006 im Rah- \\ men der organisierten Suizidbeihilfe einen verfassungsrechtlichen Anspruch \\ auf Abgabe einer tödlichen Dosis Natrium-Pentobarbital ohne Rezept.
}

\section{Christian Schwarzenegger}

Dr. iur., Assistenzprofessor für Strafrecht, Strafprozessrecht und Kriminologie an der Universität Zürich
Korrespondenz:

Dr. iur. Christian Schwarzenegger Universität Zürich

Rechtswissenschaftliches Institut Rämistrasse 74/17

CH-8001 Zürich

\section{Die Bedeutung von Natrium-Pentobar- bital für die organisierte Suizidbeihilfe}

In der organisierten Suizidbeihilfe [1] kommt seit längerer Zeit praktisch ausschliesslich NatriumPentobarbital zum Einsatz, um einer sterbewilligen Person eine risiko- und schmerzfreie Selbsttötung zu ermöglichen. Die Person nimmt in der Regel zuerst ein Mittel ein, um den später möglicherweise auftretenden Brechreiz zu unterdrücken. Danach trinkt sie etwa 60 Milliliter Wasser, in welchem eine Dosis von 10-15 Gramm Natrium-Pentobarbital aufgelöst wurde. Alternativ hierzu kann sie das Mittel auch durch eine Sonde in den Magen spritzen oder eine Kanüle öffnen, mit welcher die Lösung intravenös in den Körper gelangt. Innerhalb eines Zeitraums von 2-5 Minuten nach der Einnahme schläft die Person ein und fällt in eine tiefe Bewusstlosigkeit. Das Natrium-Pentobarbital bewirkt in der Folge eine Lähmung des Atemzentrums, was anschliessend zum Tod durch Atemstillstand führt. Die Substanz gehört zu den Barbituraten und wurde früher je nach Dosierung als Beruhigungsmittel, Schlafmittel oder Narkosemittel verwendet [2]. In der Veterinärmedizin findet es auch bei der Einschläferung von Tieren Anwendung.

Abbildung 1 zeigt, dass in der Schweiz in den letzten Jahren immer mehr Menschen diese Art von assistierter Selbsttötung gewählt haben. Rund 170-200 assistierte Suizide betreffen Personen mit Wohnsitz in der Schweiz. Stark angestiegen ist die Suizidbeihilfe zugunsten von Personen aus dem Ausland, was in der aktuellen Diskussion als «Sterbetourismus» bezeichnet wird. 59 der 76 Suizidbegleitungen, die Dignitas im Jahre 2002 durchführte, betrafen Personen, die aus dem Ausland anreisten. 2003 stieg diese Zahl auf 91 Suizide [3]. «Das Phänomen des Sterbetourismus betrifft landesweit weniger als 100 Fälle im Jahr, was 0,14\% aller Todesfälle und höchstens 7\% aller Suizide in der Schweiz ausmacht» [4].

\section{Moyens d'aide au suicide et droit à sa propre mort}

Dans le $n^{\circ}$ 11/2007 du BMS, René Raggenbass et Hanspeter Kuhn ont résumé et commenté un arrêt du Tribunal fédéral (ATF 133158 du 3 novembre 2006) sous le titre «L'aide au suicide n'est pas un droit humain». Dans cette décision de principe, le tribunal refuse un droit constitutionnel de remise d'une dose mortelle de natrium-pentobarbital sans ordonnance dans le cadre d'une aide au suicide organisé. Par la même occasion, il tient compte du fait que chaque être humain capable de discernement - même atteint de troubles psychiques - a un droit garanti par la Constitution et la Convention des droits de l'homme européenne à décider de la manière et du moment de sa propre mort. Le Prof. Schwarzenegger a formulé un commentaire sur la signification de la décision du Tribunal fédéral qui reprend I'article de MM. Raggenbass et Kuhn en mettant le doigt sur les conséquences sur le plan juridique.

\section{Die Ausgangslage}

Der vorliegende Fall betrifft eine Person, die unter einer psychischen Störung leidet, deswegen schon mehrfach stationär behandelt wurde und auch zwei Suizidversuche unternommen hatte. Weil sie das Leben mit ihrer schwer behandelbaren psychischen Störung nicht mehr als menschenwürdig erachtete, trat sie im Juli 2004 dem Verein «Dignitas» bei und bat dessen Organe im November 2004 um Suizidbeihilfe. Da für die hierzu erforderlichen 15 Gramm 
Natrium-Pentobarbital kein ärztliches Rezept erhältlich gemacht werden konnte, beantragte die Person im Sommer 2005 beim Zürcher Kantonsarzt [6] und Kantonsapotheker sowie direkt beim Bundesamt für Gesundheit (BAG), es sei ihr zu bewilligen, über den Verein «Dignitas» ohne Vorlage einer ärztlichen Verschreibung 15 Gramm dieses Stoffes zu beziehen. Begründet wurden diese Anträge vor allem damit, dass ein handlungsfähiger Mensch einen Anspruch darauf habe, sein eigenes Leben risiko- und schmerzfrei sowie ohne Gefahren für Dritte beenden zu können.

\section{Die Entscheidung des Bundesgerichts (BGE 133158)}

Nachdem alle Vorinstanzen [7] das Begehren des Suizidwilligen abgewiesen hatten, bot sich dem Bundesgericht die Möglichkeit, die gesetzlichen, verfassungs- und völkerrechtlichen Rahmenbedingungen der Abgabe von Natrium-Pentobarbital im Zusammenhang mit der organisierten Suizidbeihilfe darzulegen. Es beschäftigte sich dabei auch eingehend mit der neueren Literatur zu diesem Thema [8]. Die Verwaltungsgerichtsbeschwerde und damit die Forderung des Beschwerdeführers und des Vereins «Dignitas», die staatlichen Behörden zur Abgabe einer tödlichen Dosis Natrium-Pentobarbital ohne ärztliches Rezept zu verpflichten, wurden zu Recht abgewiesen. Es lohnt sich, die Begründung des Bundesgerichts etwas näher unter die Lupe zu nehmen.

\section{Abbildung 1}

Suizidbeihilfe in der Schweiz 1990-2005 [5].

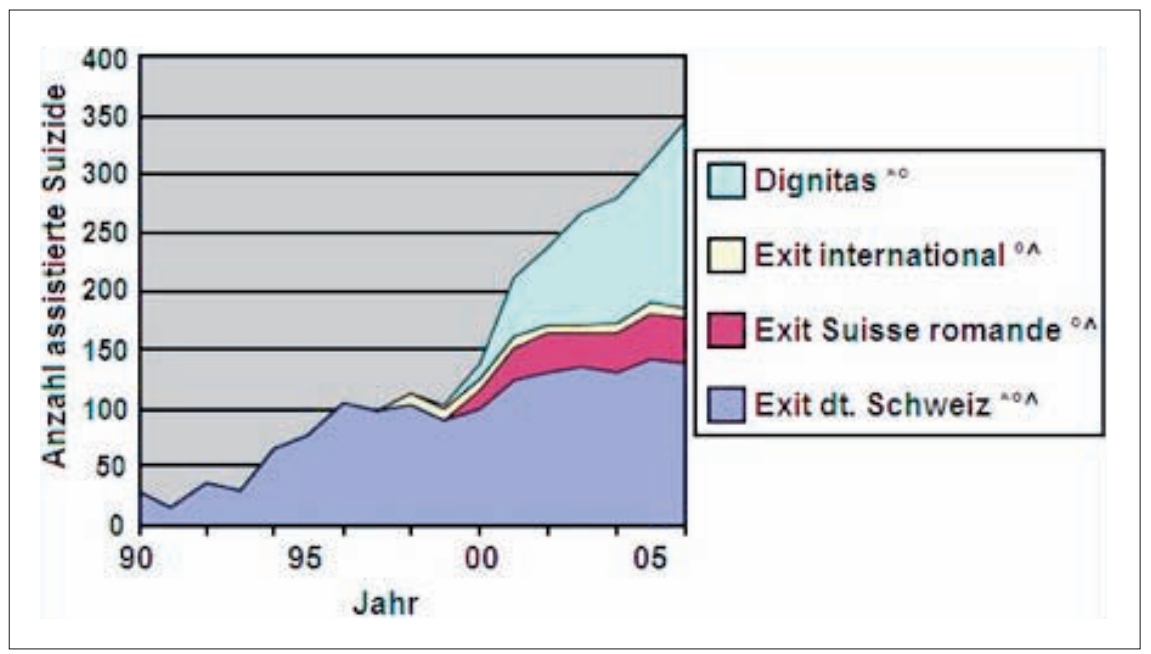

* durch das Institut für Rechtsmedizin der Universität Zürich erhoben; ${ }^{\circ}$ gemäss Angaben der Suizidbeihilfeorganisation; ^ ${ }^{\wedge}$ geschätzt.
Das anwendbare Bundesrecht verlangt grundsätzlich ein ärztliches Rezept

Natrium-Pentobarbital ist ein abhängigkeitserzeugender psychotroper Stoff und ist als solcher als Betäubungsmittel in der Betäubungsmittelverordnung verzeichnet [9]. Ausserdem dient die Substanz als Wirkstoff in einigen wenigen zugelassenen Arzneimitteln (Anästhetika). Deshalb wird es auch von der Heilmittelgesetzgebung erfasst. Anwendbar sind folglich beide: das Heilmittel- und das Betäubungsmittelgesetz (Erw. 4.1.1). Aus beiden Gesetzen ergibt sich der Grundsatz, dass Arznei- bzw. Betäubungsmittel wie Natrium-Pentobarbital nur auf ärztliches Rezept hin abgegeben werden dürfen [10]. Das Mittel darf daher nur von Ärzten und Tierärzten verschrieben werden, die aufgrund einer von einer kantonalen Behörde ausgestellten Ermächtigung zur selbständigen Berufsausübung zugelassen sind (Erw. 4.1.2). Das Heilmittelgesetz schreibt vor, dass bei der Verschreibung und der Abgabe von Arzneimitteln die anerkannten Regeln der medizinischen und pharmazeutischen Wissenschaften beachtet werden müssen [11]. Hierzu muss der Patient nach den Regeln der Kunst untersucht werden und sein Gesundheitszustand dem Arzt bekannt sein [12]. Auch Artikel 11 BetmG bestimmt, dass Ärzte Betäubungsmittel nur in dem Umfang verwenden, abgeben und verordnen dürfen, wie dies nach den anerkannten Regeln der medizinischen Wissenschaften notwendig erscheint (Erw. 4.1.2). Auch dies erfordert eine sorgfältige Diagnose, Indikationsstellung und Dokumentation sowie ein Aufklärungsgespräch. Aus den ärztlichen Berufs- und Sorgfaltspflichten - zu nennen sind insbesondere die Richtlinien der Schweizerischen Akademie der Medizinischen Wissenschaften - ergibt sich konkreter, wie ein Arzt vorzugehen hat.

Ob ein Arzt die Regeln der medizinischen oder pharmazeutischen Wissenschaften einhält, wird von den kantonalen Aufsichtsbehörden über die Ärzte überwacht. Bei Pflichtverletzungen kann die zuständige Aufsichtsbehörde, gestützt auf kantonales Gesundheitsrecht, Disziplinarmassnahmen ergreifen. Insbesondere droht dann der Entzug oder Teilentzug der Praxisbewilligung [13].

Das Bundesgericht kommt zum Schluss, dass der Beschwerdeführer nach dem geltenden Heilmittel- bzw. Betäubungsmittelrecht das von ihm gewünschte Natrium-Pentobarbital nicht erhalten könne, weil er unbestrittenermassen nicht über das notwendige ärztliche Rezept verfüge (Erw. 4.1.2 und 4.2). 
Auch das internationale Recht setzt eine ärztliche Rezeptpflicht voraus

Eine ärztliche Rezeptpflicht ergibt sich auch aus dem für die Schweiz verbindlichen internationalen Recht [14]. Nach dem Übereinkommen über psychotrope Stoffe müssen die Unterzeichnerstaaten die notwendigen Massnahmen treffen, damit die Rezepte nach den medizinischen Regeln ausgestellt und Vorschriften - insbesondere über die Wiederholbarkeit und die Gültigkeitsdauer - unterstellt werden, wie sie zum Schutz der Gesundheit und des öffentlichen Wohls erforderlich sind (Erw. 4.1.3).

Die Ausnahmeregelungen über eine Abgabe ohne ärztliches Rezept beschränken sich auf Notsituationen, die bei einer organisierten Suizidbeihilfe nicht erfüllt sind

Der Apotheker darf rezeptpflichtige Medikamente in begründeten Ausnahmefällen auch ohne ärztliche Verschreibung abgeben [15]. Wie das Bundesgericht unter Hinweis auf die Gesetzgebungsmaterialien darlegt, bezieht sich diese Bestimmung nur auf Notsituationen, also auf Fälle, in denen es zeitlich unmöglich ist, ein Rezept zu erhalten. Das kann etwa in einem Notfall notwendig werden oder zur Fortführung einer Dauertherapie, wenn das Rezept abgelaufen ist. Kein Ausnahmefall liegt aber vor, wenn ein Arzt mangels medizinischer Indikationen nicht bereit bzw. nicht in der Lage ist, ein Rezept auszustellen(sachliche Unmöglichkeit, Erw. 4.2.1).

Nach dem Betäubungsmittelrecht, das als spezielleres Recht vorgeht, darf der Apotheker in Notfällen und wenn es unmöglich ist, eine ärztliche Verschreibung rechtzeitig beizubringen, ausnahmsweise die kleinste im Handel erhältliche Packung eines Betäubungsmittels ohne Rezept abgeben. Tut er dies, muss er innert fünf Tagen der kantonalen Behörde ein Protokoll hierüber zukommen lassen und gleichzeitig den behandelnden Arzt über die Abgabe informieren [16]. Wie für die rezeptpflichtige muss auch für die rezeptfreie Abgabe nach der Betäubungsmittelverordnung eine medizinische Notwendigkeit bestehen, etwa ein akuter, lebensbedrohender Zustand eingetreten sein oder die Gefahr einer anderen gesundheitlichen Schädigung drohen. Bei einer Suizidbeihilfe wie der vom Beschwerdeführer angestrebten ist es dagegen ohne weiteres möglich und zumutbar, einen Arzt zu konsultieren, so dass auch diese Ausnahmeregelung nicht greift (Erw. 4.2.2).

Dass es sich beim Natrium-Pentobarbital um ein von der Kontrolle des BetmG teilweise ausgenommenes Betäubungsmittel handelt [17], ändert nichts an der Tatsache, dass Ärzte (und
Tierärzte) auch dieses Mittel nur für Patienten verschreiben dürfen, die sie selbst untersucht haben [18]. Da der Verein «Dignitas» keine bewilligte nationale oder internationale Organisation im Sinne des Betäubungsmittelgesetzes [19] ist, kann eine rezeptfreie Abgabe im übrigen auch nicht durch diesen erfolgen (Erw. 4.2.4).

\section{Verletzt das geltende Heilmittel-} und Betäubungsmittelrecht die von der Verfassung und der EMRK garantierten Freiheitsrechte des Suizidwilligen?

Insbesondere Ludwig A. Minelli ficht in verschiedenen Publikationen dafür [20], dass der Staat nicht nur den freiverantwortlichen Suizid tolerieren müsse, sondern dass aus der verfassungsrechtlich garantierten persönlichen Freiheit (Art. 10 Abs. 2 BV) bzw. auch aus dem konventionalrechtlich gewährleisteten Schutz des Privatlebens (Art. 8 EMRK) eine Pflicht abzuleiten sei, die Ausübung dieses Freiheitsrechts faktisch nicht durch das Heilmittel- oder Betäubungsmittelrecht zu verunmöglichen. Daraus leite sich ein Anspruch jedes Individuums - nicht nur des terminal oder sonst wie körperlich Schwerkranken - gegenüber dem Staat ab, den selbstverantwortlich beschlossenen Suizid risiko- und schmerzfrei vornehmen zu können. Der Staat müsse deshalb dafür sorgen, dass einem Suizidwilligen der Zugang zu Natrium-Pentobarbital ermöglicht werde, etwa indem gerichtlich festgehalten werde, dass ihm dieses von einem Apotheker über eine Suizidbegleitungsorganisation zur Verfügung zu stellen sei. Zudem sei der Schweizer Gesetzgeber aufgefordert, ein Verfahren einzuführen, das diesen Zugang ohne «medizinischen Paternalismus» gewährleiste, weil es auch im öffentlichen Interesse liege, die Gefahren und Schädigungen unbegleiteter Suizidversuche zu verhindern.

Das BGer lehnt diese Sichtweise ab und nimmt eine differenzierende Position ein (Erw. 6). Es bestätigt unter Hinweis auf die verfassungsrechtliche Literatur einerseits, dass zum Selbstbestimmungsrecht auch das Recht gehöre, über Art und Zeitpunkt der Beendigung des eigenen Lebens zu entscheiden; dies zumindest, soweit der Betroffene in der Lage sei, seinen entsprechenden Willen frei $\mathrm{zu}$ bilden und danach $\mathrm{zu}$ handeln (Erw. 6.1). "Vom Recht auf den eigenen Tod in diesem Sinn, das vorliegend als solches nicht in Frage gestellt ist, gilt es den vom Beschwerdeführer geltend gemachten Anspruch auf Beihilfe zum Suizid seitens des Staates oder Dritter abzugrenzen. Ein solcher lässt sich grundsätzlich weder Art. 10 Abs. 2 BV noch Art. 8 Ziff. 1 EMRK entnehmen; ein Anspruch des Sterbewilligen, dass ihm Bei- 
hilfe bei der Selbsttötung oder aktive Sterbehilfe geleistet wird, wenn er sich ausserstande sieht, seinem Leben selber ein Ende zu setzen, besteht nicht» (Erw. 6.2.1).

Aus dem Recht auf Leben [21] ergibt sich andererseits eine staatliche Pflicht, das Leben der Menschen zu schützen. Zwar geht diese Pflicht nicht so weit, dass der Staat dies auch gegen den ausdrücklichen Willen des urteilsfähigen Betroffenen selber tun müsste, «doch kann hieraus umgekehrt nicht geschlossen werden, dass er im Rahmen von Art. 8 Ziff. 1 EMRK im Sinne einer positiven Pflicht dafür zu sorgen hätte, dass ein Sterbewilliger Zugang zu einem bestimmten für den Suizid gewählten gefährlichen Stoff oder zu einem entsprechenden Instrument erhält. Das verfassungsrechtlich geschützte Recht auf Leben dürfte den Staat im vorliegenden Zusammenhang als Minimalvorgabe vorab dazu verpflichten, durch ein geeignetes Verfahren sicherzustellen, dass ein allfälliger Entscheid über die Beendigung des Lebens tatsächlich dem freien Willen des Betroffenen entspricht» (Erw. 6.2.1).

Aus der Rechtsprechung des Europäischen Gerichtshofs für Menschenrechte (EGMR) lässt sich nichts anderes ablesen (Erw. 6.2.2) [22]. Im Gegenteil: Der Gerichtshof stellt sogar fest, dass ein Staat grundsätzlich keine Handlungen billigen müsse, die den Tod eines Menschen bezweckten [23]. Es liege in erster Linie am einzelnen Staat, das Risiko und die Wahrscheinlichkeit von Missbräuchen abzuschätzen, wenn das generelle Verbot der Suizidbeihilfe gelockert oder Ausnahmen geschaffen würden; trotz möglicher Sicherungen und schützender Verfahren bestünden diesbezüglich offensichtlich Missbrauchsrisiken [24]. Ob die Tatsache, dass ein Mensch daran gehindert wird, durch die Wahl des Suizids einem Leiden zu entgehen, das er als unbillig und unwürdig empfindet, einen Eingriff in sein Recht auf Achtung des Privatlebens darstellen könnte, hat der Gerichtshof bisher offengelassen [25].

Die Selbstbestimmung und Privatsphäre sind in der Schweiz im Bereich der Suizidfreiheit nicht eingeschränkt. Auch Dritte, die ohne selbstsüchtige Beweggründe zum Suizid verleiten oder dabei Hilfe leisten, bleiben straflos [26]. Für eine wirksame Umsetzung der in Art. 8 Ziff. 1 EMRK verankerten Freiheit, über die Beendigung des eigenen Lebens entscheiden zu können, ist eine vorbehaltlose Abgabe von Natrium-Pentobarbital nicht erforderlich, auch wenn es sich für den Suizid offenbar besonders gut eignen soll (Erw. 6.2.3).

Das Bundesgericht zieht daher das Fazit, dass selbst unter der Annahme, der Schutzbereich von Art. 8 Ziff. 1 EMRK sei berührt, die mit der Rezeptpflicht für Natrium-Pentobarbital verbun- dene Beschränkung weder als verfassungs- noch als konventionswidrig gelten könnte (Erw. 6.3).

\section{Die Voraussetzungen für die Beschränkung des Selbstbestimmungsrechts des Suizidwilligen sind im vorliegenden Fall erfüllt (Art. 36 BV)}

Das Erfordernis der ärztlichen Verschreibung von Natrium-Pentobarbital beruht auf einer klar definierten gesetzlichen Grundlage [27] und ist zur Verhinderung von Straftaten bzw. der Eindämmung der mit der Suizidbeihilfe verbundenen Missbrauchsgefahren im öffentlichen Interesse erforderlich. Das Bundesgericht weist darauf hin, dass sich Missbrauchsrisiken im Zusammenhang mit der Tatherrschaft und der Eigenverantwortlichkeit des Suizidwilligen ergeben können. Auch sei die Urteilsfähigkeit ohne ärztliche Kontrolle nur schwer erstell- und nachweisbar (etwa in Abhängigkeitssituationen oder bei Beeinflussungen durch Dritte). Schliesslich könne ohne diese Kontrolle auch die Lauterkeit der Beweggründe der jeweiligen Suizidhelfer bzw. ihrer Organisation kaum geprüft werden. Die Beschränkung des Selbstbestimmungsrechts erweist sich auch als verhältnismässig bzw. als in einer demokratischen Gesellschaft notwendig. Im Rahmen der Interessenabwägung zwischen dem Schutz des Lebens - der (zumindest) gebietet, dass im Einzelfall geprüft wird, ob der Entscheid, dem Leben ein Ende zu setzen, tatsächlich dem freien und wohlerwogenen Willen des Betroffenen entspricht, falls der Sterbewillige sich für einen begleiteten Suizid mit einer der Heil- und Betäubungsmittelgesetzgebung unterstehenden Substanz entscheidet - und dem Selbstbestimmungsrecht des Betroffenen steht es der Schweiz nach der Verfassung und der Europäischen Menschenrechtskonvention frei, gewisse Voraussetzungen $\mathrm{zu}$ formulieren und in diesem Zusammenhang insbesondere etwa an der Rezeptpflicht für Natrium-Pentobarbital festzuhalten. Unter Hinweis auf Problemfälle aus der Vergangenheit sieht das Bundesgericht insbesondere keine ausreichend sichere Lösung darin, die Abgabe des Natrium-Pentobarbitals und die Verantwortung für dessen Einsatz an eine Suizidhilfeorganisation zu delegieren.

\section{Unter welchen Voraussetzungen ist eine Verschreibung von Natrium-Pentobarbital nach den anerkannten ärztlichen Berufs- und Standesregeln möglich?}

Die Zulässigkeit der Suizidbeihilfe und die Abgabe des Natrium-Pentobarbitals sind folglich an die Übereinstimmung mit den ärztlichen Berufsregeln oder den Stand der medizinischen 
Wissenschaften geknüpft. Nach den medizinisch-ethischen Richtlinien der Schweizerischen Akademie der Medizinischen Wissenschaften (SAMW) vom 25. November 2004 betreffend die Betreuung von Patientinnen und Patienten am Lebensende ist ein Gewissensentscheid des Arztes zu respektieren, wenn er sich zur Beihilfe zum Suizid entschliesst. Der Arzt trägt nach diesen Richtlinien die Verantwortung dafür, 1. dass die Erkrankung des Patienten die Annahme rechtfertigt, dass das Lebensende nahe ist; 2 . dass alternative Möglichkeiten der Hilfestellung erörtert und, soweit gewünscht, eingesetzt wurden und 3. dass der Patient sich als urteilsfähig erweist, sein Wunsch wohlerwogen und ohne äusseren Druck entstanden ist und als dauerhaft gelten kann, was durch eine unabhängige Drittperson zusätzlich überprüft werden soll, die ihrerseits nicht zwingenderweise Arzt zu sein braucht; der letzte Akt der zum Tod führenden Handlung hat immer durch den Patienten selbst zu erfolgen.

Das Bundesgericht bestätigt (Erw. 6.3.4), dass die Suizidhilfe nach neueren Auffassungen immer mehr als freiwillige ärztliche Aufgabe verstanden werde, die zwar keinem Arzt aufgedrängt werden könne, aber auch aufsichtsbzw. standesrechtlich nicht ausgeschlossen erscheine, solange bei der Untersuchung, Diagnose und Abgabe die ärztlichen Sorgfaltspflichten eingehalten werden bzw. sich der betroffene Arzt nicht hauptsächlich vom Sterbewunsch seines Patienten leiten liesse, ohne dessen Entscheid nach wissenschaftlichen Gesichtspunkten gründlich auf seine medizinische Begründetheit hin zu überprüfen.

\section{Ist Suizidbeihilfe bei Personen mit psychischen Störungen unter diesen Voraussetzungen überhaupt möglich?}

Das Bundesgericht bejaht diese besonders heikle Frage (Erw. 6.3.5). Eine unheilbare, dauerhafte, schwere psychische Beeinträchtigung kann ähnlich wie eine somatische ein Leiden begründen, das dem Patienten sein Leben auf Dauer hin nicht mehr als lebenswert erscheinen lässt. Nach neueren ethischen, rechtlichen und medizinischen Stellungnahmen ist auch in solchen Fällen eine allfällige Verschreibung von NatriumPentobarbital nicht mehr notwendigerweise kontraindiziert und generell als Verletzung der medizinischen Sorgfaltspflichten ausgeschlossen (Erw. 6.3.5.1).

Das Bundesgericht hält weiter fest, dass dabei äusserste Zurückhaltung geboten sei, weil der Sterbewunsch in diesen Fällen häufig Ausdruck einer therapierbaren psychischen Störung ist, die nach Behandlung rufe. Wenn der Sterbe- wunsch auf einer selbstbestimmten, wohlerwogenen und dauerhaften Entscheidung einer urteilsfähigen Person beruhe («Bilanzsuizid»), sei er zu respektieren. Basiere der Sterbewunsch auf einem autonomen, die Gesamtsituation erfassenden Entscheid, darf unter Umständen auch psychisch Kranken Natrium-Pentobarbital verschrieben und dadurch Suizidbeihilfe gewährt werden. Zur Feststellung der Urteilsfähigkeit ist ein vertieftes psychiatrisches Fachgutachten notwendig, was wiederum nur gewährleistet werden kann, wenn an der ärztlichen Verschreibungspflicht von Natrium-Pentobarbital festgehalten und die Verantwortung nicht (allein) in die Hände privater Suizidhilfeorganisationen gelegt wird.

\section{Die Bedeutung \\ des Bundesgerichtsentscheids}

Die Ausführungen des Bundesgerichts zum anwendbaren Bundesrecht und internationalen Recht orientieren sich am Wortsinn der Bestimmungen, an den gesetzgeberischen Motiven und den gesundheitspolizeilichen Zielsetzungen des Heilmittel- und Betäubungsmittelrechts. Sie überzeugen, denn es geht darum, Menschen (und Tiere) vor gefährlichen Heil- bzw. Betäubungsmitteln zu schützen, für einen massvollen Einsatz dieser Mittel zu sorgen und möglichst zu verhindern, dass solche Substanzen in falsche Hände geraten. Ähnlich wie beim Waffenrecht besteht das Ziel darin, den Umgang mit NatriumPentobarbital und vergleichbaren gefährlichen Substanzen auf Personen - konkret Apotheker und Ärzte - zu beschränken, die Gewähr für einen sorgfältigen Umgang bieten. Es wird leicht vergessen, dass sich die Substanz sowohl zum freiverantwortlichen Suizid als auch zur klassischen Tötung oder gar zum Mord eignet. So musste das Bundesgericht vor drei Jahren einen Fall beurteilen, in dem eine Ehefrau ihren unliebsamen Gatten mit einem Tee «zur Linderung seiner Erkältung» töten wollte. Der Trunk bestand aus Wasser, Zitrone, Honig und Natrium-Pentobarbital! Die tödliche Dosis hatte sie sich bei einem ihr unbekannten Haschischverkäufer auf der Strasse für Fr. 800.- besorgt [28]. Dass so etwas passieren kann, spricht eher für eine noch striktere Kontrolle der Natrium-PentobarbitalAbgabe und -Verfügbarkeit als für eine Lockerung der Kontrolle durch extensive Auslegung der Ausnahmebestimmungen. Die insbesondere von Frank T. Petermann vertretene Meinung, das geltende Heilmittel- und Betäubungsmittelrecht ermögliche über die Ausnahmebestimmungen unter Einhaltung weiterer Voraussetzungen eine rezeptlose Abgabe von Natrium-Pento- 
barbital bei Fällen von organisierter Suizidbeihilfe [29], überzeugt vor dem Hintergrund der erwähnten Zielsetzung des Heilmittel- und Betäubungsmittelrechts und der Missbrauchsgefahren nicht. Das Bundesgericht lehnt sie zu Recht ab. Die Verantwortlichkeit für eine sorgfaltspflichtkonforme Untersuchung des Falles würde damit ohnedies nur auf den Apotheker übertragen, denn dieser ist an die Regeln der pharmazeutischen Wissenschaft gebunden. Zudem besteht bei unsorgfältiger Abklärung der Urteilsfähigkeit, die bei Personen mit psychischen Störungen wie im vorliegenden Fall besonders heikel sein kann, sogar das Risiko, wegen fahrlässiger Tötung angeklagt $\mathrm{zu}$ werden. Schliesslich erscheint der Versuch, durch Umgehung der ärztlichen Verschreibung und der damit verbundenen Sorgfaltspflichten schneller und freier an das Natrium-Pentobarbital für die organisierte Suizidbeihilfe heranzukommen, langfristig als kontraproduktive Strategie. Die breite Akzeptanz, die die heutige Praxis der organisierten Suizidbeihilfe in der Öffentlichkeit geniesst, dürfte eben gerade darauf zurückzuführen sein, dass eine ärztliche Überprüfung im Einzelfall stattfindet. Nur eine ärztliche Mitwirkung vermag auszuschliessen, dass der Sterbewunsch z. B. im Zusammenhang mit dem Vorliegen therapierbarer oder selbstlimitierter medizinischer Krankheitsbilder steht [30]. Umgehungsstrategien dürften daher eher gesetzgeberische Initiativen auslösen, die das geltende Heilmittel- und Betäubungsmittelrecht verschärfen bzw. die organisierte Suizidbeihilfe unter eine strikte staatliche Kontrolle stellen würden.

In der Debatte über die Suizidbeihilfe werden aus politischer, ethischer und theologischer Sicht immer wieder Zweifel am Autonomieanspruch des urteilsfähigen Individuums in bezug auf seinen Tod vorgebracht. Nicht selten wird eine stärkere Durchsetzung der staatlichen Fürsorgepflicht eingefordert. Es ist daher sehr zu begrüssen, dass das Bundesgericht die letztlich massgebende Verfassungs- und Menschenrechtsordnung jetzt für den Bereich der Suizidbeihilfe genauer darlegen konnte [31]. Auf die fundamentale Bedeutung der Trennung zwischen variierenden Moralvorstellungen und der Allgemeinverbindlichkeit der Rechtsordnung im modernen demokratischen Staat hat schon Theodor Geiger in seinem Werk «Über Moral und Recht» hingewiesen [32]. Das Recht dürfe kein Instrument werden, sagt er, das durch äusseren Zwang ethische Normen bestimmter Interessengruppen durchsetze, denn dies würde eine allgemeingültige Moral voraussetzen. Doch eine solche sei in vielen Bereichen der modernen Ge- sellschaften nicht mehr auszumachen ( «Schisma der Moralen»). Werde mit dem Recht versucht, den Menschen eine Moralvorstellung aufzudrängen, untergrabe dies letztlich die Autorität des Rechts in der Gesellschaft als Ganzes. Das gilt besonders auch für die Bereiche der Sterbehilfe und Suizidbeihilfe.

Die Auslegung der Verfassung und der Europäischen Menschenrechtskonvention durch das Bundesgericht ist überzeugend. Ausgangspunkt ist der selbstbestimmte Mensch, der das Recht hat, über Art und Zeitpunkt der Beendigung des eigenen Lebens zu entscheiden, also ein «Recht auf den eigenen Tod» hat. Kann man dieses Recht auch vom Staat einfordern? Die Antwort lautet nein. Die von der Verfassung garantierte persönliche Freiheit zur Selbstbestimmung gibt dem einzelnen zwar ein Abwehrrecht gegenüber dem Staat, das ihm Schutz vor staatlichen Eingriffen und Verboten bietet. Dieses negative Recht gilt im übrigen nicht absolut. Zur Wahrung anderer Grundrechte oder öffentlicher Interessen kann es unter bestimmten Voraussetzungen eingeschränkt werden. Ein positives Recht, also eine Pflicht des Staates, dem Individuum aktiv zu einem schmerzfreien Suizid mittels Natrium-Pentobarbital zu verhelfen, ergibt sich aus dem Freiheitsrecht aber nicht. Das Bundesgericht hält dies in seiner Entscheidung explizit fest [33]. Es erteilt damit der Position Ludwig A. Minellis eine Absage. Eine andere Frage stellt sich jedoch, wenn ein Arzt freiwillig und gestützt auf eine Gewissensentscheidung Beihilfe zum Suizid leisten will. Kann ihm das in bestimmten Suizidkonstellationen durch standes- und gesundheitsrechtliche Sanktionen indirekt verboten werden? Diese Frage wird weiter unten nochmals aufgegriffen.

Nach Schweizer Recht ist das Recht auf den eigenen Tod gewährleistet. Weder durch das Strafgesetzbuch noch durch andere Gesetze wird die freiverantwortliche Selbsttötung eines urteilsfähigen Menschen beschränkt. Auch Dritte, die eine Person beim Suizid unterstützen, bleiben strafrechtlich unbehelligt, wenn sie nicht aus selbstsüchtigen Beweggründen handeln [34].

Das Bundesgericht stellt nach Klärung dieser Grundsätze aber weiter fest, dass es eine aus dem Recht auf Leben der Verfassung und der Europäischen Menschenrechtskonvention entspringende Kontrollpflicht des Staates gibt, falls beim Suizid ein gefährliches Mittel wie NatriumPentobarbital eingesetzt werden soll. Einerseits gilt es, Menschen vor übereilten Entscheidungen zu schützen; andererseits gibt es Risiken, die von der Teilnahme Dritter und insbesondere von Suizidhilfeorganisationen ausgehen (zweifelhafte 
Beweggründe der Suizidhelfer, unsorgfältige Abklärung der Wohlerwogenheit des Suizidwunsches und der Urteilsfähigkeit). Das Risiko unsorgfältiger Entscheidungen oder gar des Missbrauchs ist meines Erachtens nicht von der Hand zu weisen.

Um sicherzustellen, dass nur solchen Personen Natrium-Pentobarbital zur Selbsttötung abgegeben wird, die urteilsfähig sind und sich in Kenntnis ihres Zustandes und der alternativen Möglichkeiten sowie frei von äusserem Druck zu diesem Schritt entscheiden, muss folglich ein verlässliches Kontrollsystem eingerichtet werden. Mit der Rezeptpflicht für Natrium-Pentobarbital, die das Heilmittel- und Betäubungsmittelrecht vorsieht, garantiert der Staat, dass die erforderliche Kontrolle stattfindet. Da in der organisierten Suizidbeihilfe praktisch ausschliesslich dieses Betäubungsmittel zum Einsatz kommt, werden die Ärzte ganz zentral in den Entscheidungsprozess eingebunden. Damit rückt das Bundesgericht die Schweizer Praxis näher an ein System des ärztlich assistierten Suizids («physician-assisted suicide») heran, weil in jedem Fall eine medizinische Untersuchung erfolgen muss. Diese Klarstellung ist wichtig und begrüssenswert. Es wurde schon darauf hingewiesen, dass wichtige Abklärungen nicht ohne ärztliche Fachkenntnisse möglich sind. Ärzte bieten die beste Gewähr dafür, dass die Entscheidung für eine Suizidbeihilfe nur nach einer sorgfältigen Prüfung des Einzelfalles getroffen wird, unterstehen sie doch besonderen straf-, zivil- und gesundheitsrechtlichen Pflichten und Sanktionen. Das Bundesgericht kommt zutreffend zum Schluss, dass die Beschränkung der Verfügbarkeit von Natrium-Pentobarbital, falls sie überhaupt als Einschränkung des Selbstbestimmungsrechts angesehen werden könne, aus verfassungs- und völkerrechtlicher Sicht nicht zu beanstanden sei. Unbeantwortet bleibt allerdings die Frage, wie das (ärztliche) Kontrollsystem beim Einsatz anderer, nicht rezeptpflichtiger Stoffe wie beispielsweise Helium aussehen soll, wo die aufgezeigten Gefahren von Fehlentscheidungen oder Missbräuchen ebenfalls existieren.

Es ist meines Erachtens nicht empfehlenswert, im Bereich der organisierten Suizidbeihilfe auf andere Selbsttötungsmethoden auszuweichen, um der Rezeptpflicht zu entgehen. Dies würde unweigerlich den Druck auf die Gesetzgebung erhöhen, ein extensives Kontrollsystem über die organisierte Suizidbeihilfe zu schaffen. Es ist kaum damit zu rechnen, dass dabei auf eine ärztliche Beteiligung an den Entscheidungsprozessen verzichtet würde.
Wegen der Abhängigkeit der Suizidbeihilfe mittels Natrium-Pentobarbital von der ärztlichen Kontrolle und Rezeptausstellung kommt den ärztlichen Berufs- und Standesregeln eine zentrale Bedeutung zu [35]. Was aber entspricht den anerkannten Regeln der medizinischen Wissenschaften? Für den Bereich der Suizidbeihilfe bei Personen, deren Lebenserwartung aufgrund einer Erkrankung gering ist, ergeben sich entsprechende Sorgfaltskriterien aus den medizinisch-ethischen Richtlinien der Schweizerischen Akademie der Medizinischen Wissenschaften (SAMW) vom 25. November 2004 über die Betreuung von Patientinnen und Patienten am Lebensende. Die Bereitschaft des Arztes, einer Person einen begleiteten Suizid zu ermöglichen, wird als Gewissensentscheid explizit anerkannt. Die Verschreibung von Natrium-Pentobarbital wird von klar definierten Bedingungen abhängig gemacht. Diese werden auch vom Bundesgericht bekräftigt.

Für die Fallkonstellationen der Suizidbeihilfe bei Personen, die nicht unmittelbar in Todesnähe stehen, existieren keine expliziten Berufs- und Standesregeln der medizinischen Wissenschaften. Dies bedeutet jedoch nicht, wie es der Zürcher Kantonsarzt in einem Rundschreiben an die Ärzteschaft des Kantons Zürich im September 1999 festhält, dass eine Verschreibung von Natrium-Pentobarbital in all diesen Fällen einem Verstoss gegen das Berufsrecht der Ärzte gleichkommen würde und zu gesundheitsrechtlichen Sanktionen führen müsse. Dies würde etwa den Personen mit psychischen Störungen faktisch das Selbstbestimmungsrecht verweigern. In einem Gutachten zuhanden von Exit [36] wird eine Lösung aufgezeigt, unter welchen Voraussetzungen ein Arzt auch einer Person mit psychischen Störungen ein Rezept ausstellen darf. In den zahlenmässig seltenen Fällen urteilsfähiger Personen mit psychischer Störung kann - ähnlich wie dies bei Patienten in einem chronisch vegetativen $\mathrm{Zu}$ stand ohne Todesnähe zur Rechtfertigung des Abbruchs lebenserhaltender Massnahmen herangezogen wird - aufgrund einer sorgfältigen Abklärung, die der negativen Verlaufsprognose, dem Leidenszustand des Patienten und der Dauerhaftigkeit dieses Leidens Rechnung trägt, eine Verschreibung von Natrium-Pentobarbital in tödlicher Dosis als medizinisch indiziert und damit statthaft angesehen werden. Die Bestimmung der Urteilsfähigkeit erweist sich allerdings bei dieser Personengruppe als besonders schwierig, weshalb ein psychiatrisches Gutachten hierüber unverzichtbar ist. Dass sich das Verwaltungsgericht des Kantons Zürich [37] und jetzt 
auch das Bundesgericht diesem Lösungsvorschlag anschliessen, ist von besonderer Bedeutung, weil er für die Ärzteschaft mehr Rechtssicherheit schafft. Hält sich der Arzt bei der Rezeptausstellung an die genannten Sorgfaltskriterien, hat er nicht mit straf-, zivil- und gesundheitsrechtlichen Sanktionen zu rechnen.

$\mathrm{Zu}$ wünschen wäre allerdings, dass sich die Schweizerische Akademie der Medizinischen Wissenschaften nochmals mit dem Thema der ärztlichen Beteiligung an begleiteten Suiziden auseinandersetzen würde, um ihre Standesregeln zu ergänzen. Gerade weil das Recht und die Rechtsprechung des Bundesgerichts den Ärzten eine zentrale Kontrollfunktion übertragen, kann sich die Ärzteschaft nicht in ein «Schweigen» flüchten. Die Realität der Suizidbeihilfe betrifft auch Personen, deren Lebenserwartung (noch) nicht gering ist, deren Suizidwunsch aus einer Reflexion über ihre psychischen Störungen oder ihre sich verschlechternden kortikalen Funktionen (Demenz- und Alzheimerpatienten) entstammt. Nicht wenige Ärzte wollen auch in diesen Fällen Gewissensentscheide treffen können, und diese Entscheidungen sollten durch standesrechtliche Richtlinien angeleitet sein. Bleibt die medizinische Wissenschaft inaktiv, müsste die Bestimmung von Sorgfaltskriterien im Umgang mit solchen Fällen allein durch die Rechtsprechung entwickelt werden.

\section{Literatur}

1 Derzeit leisten vier Vereine in organisierter Form Suizidbeihilfe. Es sind dies Exit - Deutsche Schweiz (www.exit.ch), Exit A.D.M.D. Suisse romande (www.exit-geneve.ch), Ex international (www.exinternational.ch) und Dignitas (www.dignitas.ch)

2 Vgl. zu dieser Suizidmethode Ludwig A. Minelli, Die EMRK schützt die Suizidfreiheit. Wie antwortet darauf das Schweizer Recht? AJP 2004, 491-504, $496 \mathrm{f}$.

3 Bundesamt für Gesundheit, Suizid und Suizidprävention in der Schweiz, Bericht in Erfüllung des Postulats Widmer (02.3251), o.O. [Bern] 2005, 16.

4 Eidgenössisches Justiz- und Polizeidepartement, Sterbehilfe und Palliativmedizin - Handlungsbedarf für den Bund?, Bern 2006, 49.

5 Quelle: Georg Bosshard, Empfehlungen der Arbeitsgruppe «Suizidbeihilfe und Spital», Vortrag am Universitätsspital Zürich 8.3.2007.

6 Aus Gründen der einfacheren Lesbarkeit wird auf die geschlechtsspezifische Differenzierung verzichtet. Entsprechende Begriffe gelten im Sinne der Gleichbehandlung grundsätzlich für beide Geschlechter.

7 Vgl. insbesondere VerwGer ZH, 17.11.2005, VB.2005.00345.
8 Neben dem obenerwähnten Aufsatz von

L. A. Minelli [2] sind insbesondere zu nennen: Tobias Jaag/Markus Rüssli, Sterbehilfe in staatlichen Spitälern, Kranken- und Altersheimen, ZBl 2001, 113-129; Georg Bosshard/Walter Bär Sterbeassistenz und die Rolle des Arztes. Überlegungen zur aktuellen Debatte um die Regelung von Suizidbeihilfe und aktiver Sterbehilfe in der Schweiz, AJP 2002, 407-413; Klaus Peter Rippe/ Christian Schwarzenegger/Georg Bosshard/Martin Kiesewetter, Urteilsfähigkeit von Menschen mit psychischen Störungen und Suizidbeihilfe, SJZ 2005, 53-62 und 81-91; Eidgenössisches Justizund Polizeidepartement, Sterbehilfe und Palliativmedizin - Handlungsbedarf für den Bund?, Bern 2006; Frank T. Petermann, Rechtliche Überlegungen zur Problematik der Rezeptierung und Verfügbarkeit von Natrium-Pentobarbital, AJP 2006, 439-467; Claude Rouiller/Leila Roussianos, Le droit à la vie et le droit de mourir dignement, in: Jusletter 12. Juni 2006, N 1-111 (abrufbar unter: www.weblaw.ch). Vgl. auch Christian Schwarzenegger, in: Marcel A. Niggli/Hans Wiprächtiger (Hrsg.), Basler Kommentar Strafgesetzbuch II, Basel 2003, vor Art. 111 N 3 ff. und N 20 ff.; Art. 115 N 1 ff. mit zahlreichen Nachweisen (2. Aufl., Basel 2007 in Vorbereitung)

9 Art. 1 Abs. 2 lit. c i.V.m. Art. 1 Abs. 4 BetmG; Art. 1 Abs. 1 und Art. 2 Abs. 1 BetmV-Swissmedic.

10 Art. 24 Abs. 1 lit. a HMG; Art. 10 i.V.m. Art. 9 Abs. 1 BetmG.

11 Art. 26 Abs. 1 HMG.

12 Art. 26 Abs. 2 HMG.

13 Vgl. dazu näher Rippe/Schwarzenegger/Bosshard/ Kiesewetter [8] $87 \mathrm{ff}$.

14 Liste III des Übereinkommens vom 21. Februar 1971 über psychotrope Stoffe [SR 0.812.121.02], Art. 9 Ziff. 1.

15 Art. 24 Abs. 1 lit. a HMG.

16 Art. 48 Abs. 1-2 BetmV.

17 Vgl. Art. 3 lit. b BetmV i.V.m. mit Art. 2 Abs. 1 und Anhang b BetmV-Swissmedic.

18 Art. 43 Abs. 1 BetmV.

19 Art. 14a BetmG.

20 Minelli [2] 491 ff., dort weitere Nachweise.

21 Art. 10 Abs. 1 BV, Art. 2 EMRK.

22 EGMR, Pretty v. United Kingdom [Appl. no. 2346/02], 29. April 2002, § 40; deutsche Übersetzung in: NJW 2002, 2851 ff.

23 EGMR, Pretty v. United Kingdom [Appl. no. 2346/02], 29. April 2002, § 55.

24 EGMR, Pretty v. United Kingdom [Appl. no. 2346/02], 29. April 2002, §§ 74-78.

25 EGMR, Pretty v. United Kingdom [Appl. no. 2346/02], 29. April 2002, § 67.

26 Vgl. Art. 115 StGB.

27 Verschreibungspflicht, siehe Art. 24, 26 HMG; Art. 9, 10 Abs. 1 BetmG; Art. 9 Abs. 1 und Tabelle 3 des Übereinkommens über psychotrope Stoffe.

28 BGE 6S.10/2004 vom 1. April 2004 
29 Petermann [8] 463 ff.

30 Bosshard/Bär [8] $412 \mathrm{f}$.

31 Vgl. mit spezifischem Fokus auf Suizidbeihilfe: Marco Borghi, L'art. 115 CP fra rispetto dell'autonomia individuale e esigenza di regolamentazione. Rivista ticinese di diritto 1/2005, 555-578; Schwarzenegger [8] vor Art. $111 \mathrm{~N} 3$ ff. und N 20 ff.; Art. 115 N 1 ff.

32 Theodor Geiger, Über Moral und Recht, Berlin 1979 (Orig. 1945), 182 ff.

33 Es besteht folglich auch kein grundrechtlicher Anspruch auf ärztliche Suizidbeihilfe, wie Raggenbass/Kuhn zu Recht schreiben.
34 Dazu näher Schwarzenegger [8] vor Art. 111 N 3 ff.; Art. 115 N 1 ff.

35 Frank T. Petermann hat sich intensiv mit diesen Berufs- und Standesregeln auseinandergesetzt, siehe Petermann [8] 448 ff.

36 Rippe/Schwarzenegger/Bosshard/Kiesewetter [8] $90 \mathrm{f}$.

37 VerwGer ZH, 17.11.2005, VB.2005.00345, Erw. 3.6.2.

\section{L'ASSM donnera suite à la suggestion de l'auteur (voir ci-dessus) et prendra position dans un des prochains numéros du BMS.}

